\title{
SOLUTIONS OF FOURTH-ORDER EVOLUTION EQUATIONS IN MATERIAL SCIENCE
}

\author{
DILRUK GALLAGE
}

(Received 19 June 2020; first published online 5 October 2020)

2020 Mathematics subject classification: primary 35K55; secondary 35K57.

Keywords and phrases: fourth-order diffusion, inverse method, similarity solutions, nonlinear PDE, Allen-Cahn equation, Cahn-Hilliard equation, nonlinear reaction-diffusion.

Two classes of fourth-order nonlinear partial differential equations are considered. One of them plays a vital role in understanding the surface redistribution of axisymmetric crystalline materials due mainly to surface diffusion, while the other makes a significant contribution in the modelling of the process of phase separation of a binary-component system near the critical temperature.

Firstly, by extending an inverse method previously used for the second-order one-dimensional nonlinear diffusion equation, we construct similarity solutions to a fourth-order nonlinear partial differential equation for axisymmetric surface diffusion. By imposing a solution profile, both a feasible surface tension and an effectively related mobility function are deduced simultaneously. An optimisation algorithm is implemented to construct a well-defined mobility function that is effectively a single-valued function of surface orientation. It is shown that the solution of the linear model well approximates the solution of the nonlinear model, in which the surface tension and mobility are close to constant for a wide range of surface angles, even when nonlinear geometric terms are included.

Lastly, using the nonclassical symmetry of nonlinear reaction-diffusion equations, some exact multi-dimensional time-dependent solutions are constructed for a fourth-order Allen-Cahn-Hilliard equation. A phase field which provides a phenomenological description of a two-phase system near critical temperature is modelled by the fourth-order Allen-Cahn-Hilliard equation. We obtain solutions for the changing phase of a cylindrical or spherical inclusion, which allow for a 'mushy' zone with a mixed state that is controlled by imposing a pure state at the boundary. Since the transport properties of the mixture depend upon the relative proportions of the two phases, the diffusion coefficients for the transport of one phase through the

Thesis submitted to La Trobe University in January 2020; degree approved on 12 February 2020; principal supervisor Philip Broadbridge, cosupervisors Dimetre Triadis and Pierluigi Cesana.

(C) 2020 Australian Mathematical Publishing Association Inc. 
mixture depend upon the phase field value. A source term provides stability of both the pure phases; however, this behaviour may be controlled or even reversed through the boundary conditions.

Parts of this research have been published in [1,2].

\section{References}

[1] P. Broadbridge, D. Triadis, D. Gallage and P. Cesana, 'Nonclassical symmetry solutions for fourth-order phase field reaction-diffusion', Symmetry 10(3) (2018), Article ID 72.

[2] D. Gallage, D. Triadis, P. Broadbridge and P. Cesana, 'Solution for 4th-order nonlinear axisymmetric surface diffusion by inverse method', Phys. D 405 (2020), Article ID 132288.

DILRUK GALLAGE, Department of Mathematics, University of Colombo, Colombo 00700, Sri Lanka

e-mail: dilruk@sci.cmb.ac.lk 